\title{
Neurobiology of Behavior
}

\section{Citation}

Dulac, Catherine, and Giacomo Rizzolatti. 2009. Neurobiology of behavior. Current Opinion in Neurobiology 19(6): 634-636.

\section{Published Version}

doi:10.1016/j.conb.2009.10.009

\section{Permanent link}

http://nrs.harvard.edu/urn-3:HUL.InstRepos:9709718

\section{Terms of Use}

This article was downloaded from Harvard University's DASH repository, and is made available under the terms and conditions applicable to Open Access Policy Articles, as set forth at http:// nrs.harvard.edu/urn-3:HUL.InstRepos:dash.current.terms-of-use\#OAP

\section{Share Your Story}

The Harvard community has made this article openly available.

Please share how this access benefits you. Submit a story.

\section{Accessibility}




\section{Neurobiology of behavior}

Catherine Dulac

Howard Hughes Medical Institute, Department of Molecular and Cellular Biology, Harvard University, 16 Divinity Avenue, Cambridge, MA 02138, United States

\section{Giacomo Rizzolatti}

Dipartimento di Neuroscienze, Sezione di Fisiologia, Università di Parma, Via Volturno, 9/E, 43100 Parma, Italy 
Introduction

Behavior is a broad term that covers a variety of largely different activities. It describes 'simple behaviors' mediated by neuronal networks of 'simple' model systems, as well as moral or economic decisions mediated by the complex circuits of the human brain. The biological unity between a fish (or even a worm like Caenorhabditis elegans) and individuals making economical or moral decisions might sound outrageous to people who (still) think that neurosciences describe the 'hardware' of the brain and cannot 'invade' fields like aesthetics or economics historically reserved to nonbiological disciplines. The evidence (see this issue) is just the opposite. Of course neuroscience is able to explain much better behaviors underpinned by 'simple' model systems, but the neurophysiological knowledge acquired in the last years on discipline considered outside neuroscience is impressive. This issue will show on the one hand the similarity of neurophysiological mechanisms underlying behavior of brain circuits with various degrees of complexity, on the other the enormous progresses achieved in these last years on the neural basis of fields considered outside the neuroscientific enquiry.

Model systems

Technological and analytical advances in the behavioral studies of vertebrate and invertebrate model systems are contributing to an emerging understanding of the mechanistic operations performed by identified neuronal assemblies and leading to measurable behavioral responses. Three of the reviews in this issue explore how multilevel analysis developed recently in the nematode, fish, and song bird, offer amenable paradigms for a systems-level understanding of microcircuit activity and behavior. A fourth review covers the astonishing diversity of sexdetermination in fish, opening new avenues for a genetic underpinning of differences in male versus female sex and social behaviors.

The paper by Sengupta and Samuel reviews how quantitative algorithms are well suited to describe the biomechanics of $\mathrm{C}$. elegans locomotor activity and to dissect the contribution of individual neurons and of larger neuronal assemblies to the control of motor and sensory activity. This, together with the use of optogenetic tools has led to the development of powerful new assays to monitor and manipulate neuronal activity patterns in the living behaving transparent nematode. The fast pace of recent publications in the field led the authors to anticipate how refined microstimulations of increasingly smaller and more defined neuronal assemblies will 
enable precise identification of the neuronal nodes of sensory and motor control, and a more mechanistic understanding of neuronal computations required by specific behavioral metrics. In their manuscript, Portuges and Engert argue that behavior analyses of the larval zebrafish offer the remarkable advantage of studying a behaving transparent organism in a vertebrate context. The authors review four behavioral paradigms, from the simple and robust optokinetic reflex and optomotor response, to the more elaborate prey tracking and capture, and the visual startle and escape response. In these tractable behavioral paradigms, distinct patterns of visual stimulation lead to well defined and measurable behavioral outputs, while activity of associated neuronal networks can be directly visualized and altered in living animals, thanks to improved imaging and optogenetic approaches. As seen with C. elegans, although not as detailed yet, a number of new publications aim at identifying the parameters and metrics of neuronal activity patterns associated with specific locomotor behaviors.

The paper by Desjardins and Fernald explores the evolution of sex-differences in behavior. Fish, which represent the largest group of vertebrates, display an astonishing variety of physiological and neural mechanisms underlying sex determination and sex-specific behaviors. Reviewing the literature on teleost fish, the authors describe the evolution and variation in neural pathways underlying reproductive systems, and lay the foundations for genetic and epigenetic mechanisms that may provide the regulatory templates for this remarkable variability.

In a fourth review Richard Mooney tackles the complex cognitive issues underlying the neurobiology of song learning in the juvenile male zebra finch and discusses recent progress in a model system of imitative learning that share similarities with human speech learning. The author describes how interconnected brain nuclei are specialized for controlling and coordinating the tasks of singing and of learning a song from the male tutor. Interestingly, it appears that neuronal responses in nuclei participating in these circuits are characterized by distinct sparseness and rhythmic patterns of activity. Recent insights into the control and variability of these activity patterns are providing important clues about the central mechanisms for auditory feedback, song memory, and plasticity, which enable the juvenile bird to compare and adapt its own song to the memorized template of the tutor song.

Higher functions

The discovery of mirror neurons opened a new era in the study of the neural basis of imitation. The early mirror neuron studies on this topic defined the 'core' neural circuit for imitation, a 
circuit that includes the posterior part of the superior temporal sulcus, the inferior parietal lobule, and the posterior part of the inferior frontal gyrus plus the adjacent ventral premotor cortex. The mirror mechanism located in the areas of this circuit maps the observed motor acts onto similar motor representations in the observer's brain, allowing in this way their imitation. In recent years the study of neurobiology of imitation rapidly expanded. In his review Iacoboni examines these new findings. He discussed the mechanisms that, in addition to the core mechanism, are necessary for imitation learning, the control mechanism that prevents undesired overt imitation and, finally, as the developmental trajectory of neural mechanism of imitation. This exhaustive and balanced review ends with a discussion of vocal learning in birds and its possible relations with similar mechanisms of vocal learning in humans.

Mirror neurons are also on the core of the review by Keysers and Gazzola on the neural basis of sharing. These authors expand the classical interpretation of mirror neurons as a mechanism for understanding motor acts. Their view is that mirror mechanism is an aspect of a larger 'vicarious' system that allows us to share actions, emotions and sensations of others. They first review recent data on the mirror mechanism for action suggesting that mirror mechanism is present in various other cortical areas besides the classical parietal-premotor circuit. They then discuss the 'vicarious' somatosensory, and emotional activity. While for emotional sharing a motor mechanism similar to that of actions sharing is easy to postulate, this is not the case for sharing sensation. Keysers and Gazzola make an attempt to explain it, suggesting a possible neural pathway. This attempt is interesting; although, for a firm conclusion more empirical data are needed. The review includes two excellent boxes: one on 'what' is mirrored in the motor system, and one on how we 'get to have mirror neurons'.

The next review concerns neuroeconomics. While in the two previous reviews the concepts borrowed from other disciplines are usually well known to cognitive neuroscience researchers, this is not always the case for economics. Thus, Rustichini explains first some basic notions of economics and, in particular, theories of decision-making. Establishing a basic conceptual framework, he presents neural data on decision-making and discusses how these data fit the economic theories. This interdisciplinary operation, although undoubtedly difficult, was very successful and Rustichini's review provides an excellent example on the utility of the 'intrusion' of neuroscience in a nonbiological discipline. The transition from a two-dimensional to a fivedimensional view of human decision-making might be still tentative, but it is clear from the 
available evidence that this enriched view of economic decisions goes in the right direction and part of the merit is of the neural approach.

I am pretty sure that most readers, but those directly involved in the field, will be impressed by the tremendous progress that has been achieved in the effort to understand the neural basis of human morality. The picture that emerges from the review by Funk and Gazzaniga points out toward a model in which automatic processing in parallel neural circuits, many of which are associated with social emotions, evaluate the action and intentions of others. Through competition, a subset of these circuits wins and ultimately causes a decision. The authors argue that, at the end, an interpretative process offers a post hoc explanation of the decision that is experienced consciously as a subjective moral sense of right or wrong. Maybe not everybody would agree with this last conclusion. The review, however, is so well written and well argued, that they also will enjoy reading it.

The final review of this issue concerns Neuroesthetics. Probably this is the most difficult field to approach with neuroscience techniques among those we dealt with in this issue. The main reason is that the basic concepts of aesthetics, starting from the most fundamental ones, are often ambiguous. What does beauty mean? Is beauty something objective that has a biological basis, or is it a mere subjective feeling? How much cultural pressure or even present day fashion determines what we call aesthetic experience? Unfortunately, most studies in Neuroesthetics avoided these basic questions, asking individuals (very simplistically) whether or not they like some works of art. By doing that they mixed the issue of the possible existence of an objective, biologically determined experience of beauty, cognitive reasoning on whether an object reaches some subjective standard, and, finally, emotional factors that have nothing to do with beauty (a perfectly ugly painting can be liked for mere sentimental reasons). By mixing all these factors in a single experimental design, it is not surprising that, as noted by Di Dio and Gallese, the achieved neural results are heterogeneous. In spite of this there is evidence that some brain centers are frequently activated in Neuroesthetics experiments. These centers are the sensorimotor cortex, the core emotional areas and the reward-related centers. On the basis of these findings, the authors propose that aesthetic experience is a 'multilevel process exceeding a purely visual analysis of artworks, and heavily relying upon visceromotor and somatomotor resonance in the beholder'. This conclusion is very reasonable. However, it also shows that a lot 
of work remains to be done for specifying the actual roles of the different centers mentioned and also for a better definition of concepts derived from aesthetics. 